$\mathrm{J}$ o u r n a l of

Mathematics

and Applications

JMA No 40, pp 85-96 (2017)

\title{
Weak Solutions of Fractional Order Differential Equations via Volterra-Stieltjes Integral Operator
}

\author{
Ahmed M.A El-Sayed, Wagdy G. El-Sayed \\ and A.A.H. Abd El-Mowla
}

\begin{abstract}
The fractional derivative of the Riemann-Liouville and Caputo types played an important role in the development of the theory of fractional derivatives, integrals and for its applications in pure mathematics ([18], [21]). In this paper, we study the existence of weak solutions for fractional differential equations of Riemann-Liouville and Caputo types. We depend on converting of the mentioned equations to the form of functional integral equations of Volterra-Stieltjes type in reflexive Banach spaces.
\end{abstract}

AMS Subject Classification: 35D30, 34A08, 26A42.

Keywords and Phrases: Weak solution; Mild solution; Weakly Riemann-Stieltjes integral; Function of bounded variation.

\section{Introduction and preliminaries}

Let $E$ be a reflexive Banach space with norm $\|$. $\|$ and dual $E^{*}$. Denote by $C[I, E]$ the Banach space of strongly continuous functions $x: I \rightarrow E$ with sup-norm.

Fractional differential equations have received increasing attention due to its applications in physics, chemistry, materials, engineering, biology, finance [15, 16]. Fractional order derivatives have the memory property and can describe many phenomena that integer order derivatives cant characterize. Only a few papers consider fractional differential equations in reflexive Banach spaces with the weak topology $[6,7,14,22$, 23].

Here we study the existence of weak solutions of the Volterra-Stieltjes integral equation

$$
x(t)=p(t)+\int_{0}^{t} f(s, x(s)) d_{s} g(t, s), \quad t \in I=[0, T],
$$

COPYRIGHT @ by Publishing House of Rzeszów University of Technology P.O. Box 85, 35-959 Rzeszów, Poland 
in the reflexive Banach space $E$.

Let $\alpha \in(0,1)$. As applications, we study the existence of weak solution for the differential equations of fractional order

$$
{ }^{R} D^{\alpha} x(t)=f(t, x(t)), t \in(0, T]
$$

with the initial data

$$
x(0)=0,
$$

where ${ }^{R} D^{\alpha} x($.$) is a Riemann-Liouville fractional derivative of the function x: I=$ $[0, T] \rightarrow E$.

Also we study the existence of mild solution for the initial value problem

$$
{ }^{C} D^{\alpha} x(t)=f(t, x(t)), t \in(0, T]
$$

with the initial data

$$
x(0)=x_{0},
$$

where ${ }^{C} D^{\alpha} x($.$) is a Caputo fractional derivative of the function x: I:[0, T] \rightarrow E$. Functional integral equations of Volterra-Stieltjes type have been studied in the space of continuous functions in many papers for example, (see [1-5] and [8]).

For the properties of the Stieltjes integral (see Banaś [1]).

Definition 1.1. The fractional (arbitrary) order integral of the function $f \in L_{1}$ of order $\alpha>0$ is defined as [18, 21]

$$
I^{\alpha} f(t):=\int_{0}^{t} \frac{(t-s)^{\alpha-1}}{\Gamma(\alpha)} f(s) d s .
$$

For the fractional-order derivative we have the following two definitions.

Definition 1.2. The Riemann-Liouville fractional-order derivative of $f(t)$ of order $\alpha \in(0,1)$ is defined as ([18], [21])

$$
{ }^{R} D_{a}^{\alpha} f(t)=\frac{d}{d t} \int_{a}^{t} \frac{(t-s)^{-\alpha}}{\Gamma(1-\alpha)} f(s) d s
$$

or

$$
{ }^{R} D_{a}^{\alpha} f(t)=\frac{d}{d t} I_{a}^{1-\alpha} f(t) .
$$

Definition 1.3. The Caputo fractional-order derivative of $g(t)$ of order $\alpha \in(0,1]$ of the absolutely continuous function $g(t)$ is defined as $([9])$

$$
{ }^{C} D_{a}^{\alpha} g(t)=\int_{a}^{t} \frac{(t-s)^{-\alpha}}{\Gamma(1-\alpha)} \frac{d}{d s} g(s) d s
$$

or

$$
{ }^{C} D_{a}^{\alpha} g(t)=I_{a}^{1-\alpha} \frac{d}{d t} g(t) .
$$


Now, we shall present some auxiliary results that will be need in this work. Let $E$ be a Banach space (need not be reflexive) and let $x:[a, b] \rightarrow E$, then

(1-) $x($.$) is said to be weakly continuous (measurable) at t_{0} \in[a, b]$ if for every $\phi \in E^{*}, \phi(x()$.$) is continuous (measurable) at t_{0}$.

(2-) A function $h: E \rightarrow E$ is said to be weakly sequentially continuous if h maps weakly convergent sequences in $E$ to weakly convergent sequences in $E$.

If $x$ is weakly continuous on $I$, then $x$ is strongly measurable and hence weakly measurable (see [10] and [13]). It is evident that in reflexive Banach spaces, if $x$ is weakly continuous function on $[a, b]$, then $x$ is weakly Riemann integrable (see [13]).

Definition 1.4. Let $f: I \times E \rightarrow E$. Then $f(t, u)$ is said to be weakly-weakly continuous at $\left(t_{0}, u_{0}\right)$ if given $\epsilon>0, \phi \in E^{*}$ there exists $\delta>0$ and a weakly open set $U$ containing $u_{0}$ such that

$$
\left|\phi\left(f(t, u)-f\left(t_{0}, u_{0}\right)\right)\right|<\epsilon
$$

whenever

$$
\left|t-t_{0}\right|<\delta \text { and } u \in U \text {. }
$$

Now, we have the following fixed point theorem, due to O'Regan, in the reflexive Banach space (see [19]) and some propositions which will be used in the sequel [13, $20]$.

Theorem 1.5. Let $E$ be a Banach space and let $Q$ be a nonempty, bounded, closed and convex subset of $C[I, E]$ and let $F: Q \rightarrow Q$ be a weakly sequentially continuous and assume that $F Q(t)$ is relatively weakly compact in $E$ for each $t \in I$. Then, $F$ has a fixed point in the set $Q$.

Proposition 1.6. A convex subset of a normed space $E$ is closed if and only if it is weakly closed.

Proposition 1.7. A subset of a reflexive Banach space is weakly compact if and only if it is closed in the weak topology and bounded in the norm topology.

Proposition 1.8. Let $E$ be a normed space with $y \in E$ and $y \neq 0$. Then there exists $a \phi \in E^{*}$ with $\|\phi\|=1$ and $\|y\|=\phi(y)$.

\section{Volterra-Stieltjes integral equation}

In this section we prove the existence of weak solutions for the Volterra-Stieltjes integral equation

$$
x(t)=p(t)+\int_{0}^{t} f(s, x(s)) d_{s} g(t, s), \quad t \in I=[0, T],
$$


in the space $C[I, E]$. To facilitate our discussion, denote $\Lambda$ by

$$
\Lambda=\{(t, s): 0 \leq s \leq t \leq T\}
$$

and let $p: I \rightarrow E, f: I \times E \rightarrow E$ and $g: \Lambda \rightarrow R$ be functions such that:

(i) $p \in C[I, E]$.

(ii) The function $f$ is weakly-weakly continuous.

(iii) There exists a constant $M$ such that $\|f(t, x)\| \leq M$.

(iv) The function $g$ is continuous on $\Lambda$.

(v) The function $s \rightarrow g(t, s)$ is of bounded variation on $[0, t]$ for each fixed $t \in I$.

(vi) For any $\epsilon>0$ there exists $\delta>0$ for all $t_{1}, t_{2} \in I$ such that $t_{1}<t_{2}$ and $t_{2}-t_{1} \leq$ $\delta$ the following inequality holds

$$
\bigvee_{0}^{t_{1}}\left[g\left(t_{2}, s\right)-g\left(t_{1}, s\right)\right] \leq \epsilon
$$

(vii) $g(t, 0)=0$ for any $t \in I$.

Obviously we will assume that $g$ satisfies assumptions (iv)-(vi). For our purposes we will only need the following lemmas.

Lemma 2.1. [5] The function $z \rightarrow \bigvee_{s=0}^{z} g(t, s)$ is continuous on $[0, t]$ for any fixed $t \in$ $I$.

Lemma 2.2. [5] For an arbitrary fixed $0<t_{2} \in I$ and for any $\epsilon>0$, there exists $\delta>$ 0 such that if $t_{1} \in I, t_{1}<t_{2}$ and $t_{2}-t_{1} \leq \delta$ then

$$
\bigvee_{s=t_{1}}^{t_{2}} g\left(t_{2}, s\right) \leq \epsilon
$$

Lemma 2.3. [5] The function $t \rightarrow \bigvee_{s=0}^{t} g(t, s)$ is continuous on I. Then there exists a finite positive constant $K$ such that

$$
K=\sup \left\{\bigvee_{s=0}^{t} g(t, s): t \in I\right\} .
$$

Definition 2.4. By a weak solution to (2.5) we mean a function $x \in C[I, E]$ which satisfies the integral equation (2.5). This is equivalent to find $x \in C[I, E]$ with

$$
\phi(x(t))=\phi\left(p(t)+\int_{0}^{t} f(s, x(s)) d_{s} g(t, s)\right), t \in I \forall \phi \in E^{*} .
$$


Now we can prove the following theorem.

Theorem 2.5. Under the assumptions (i)-(vii), the Volterra-Stieltjes integral equation (2.5) has at least one weak solution $x \in C[I, E]$.

Proof. Define the nonlinear Volterra-Stieltjes integral operator $A$ by

$$
A x(t)=p(t)+\int_{0}^{t} f(s, x(s)) d_{s} g(t, s), \quad t \in I .
$$

For every $x \in C[I, E], f(., x()$.$) is weakly continuous ([24]). To see this we$ equip $E$ and $I \times E$ with weak topology and note that $t \mapsto(t, x(t))$ is continuous as a mapping from $I$ into $I \times E$, then $f(., x()$.$) is a composition of this mapping$ with $f$ and thus for each weakly continuous $x: I \rightarrow E, f(., x()):. I \rightarrow E$ is weakly continuous, means that $\phi(f(., x())$.$) is continuous, for every \phi \in E^{*}, g$ is of bounded variation. Hence $f(., x()$.$) is weakly Riemann-Stieltjes integrable on I$ with respect to $s \rightarrow g(t, s)$. Thus $A$ makes sense.

For notational purposes $\|x\|_{0}=\sup _{t \in I}\|x(t)\|$.

Now, define the set $Q$ by

$$
\begin{gathered}
Q=\left\{x \in C[I, E]:\|x\|_{0} \leq M_{0}\right. \\
\left.\left\|x\left(t_{2}\right)-x\left(t_{1}\right)\right\| \leq\left\|p\left(t_{2}\right)-p\left(t_{1}\right)\right\|+M N(\epsilon)+M \bigvee_{s=t_{1}}^{t_{2}} g\left(t_{2}, s\right)\right\} .
\end{gathered}
$$

First notice that $Q$ is convex and norm closed. Hence $Q$ is weakly closed by Proposition 1.6.

Note that $A$ is well defined, to see that, Let $t_{1}, t_{2} \in I, t_{2}>t_{1}$, without loss of generality, assume $A x\left(t_{2}\right)-A x\left(t_{1}\right) \neq 0$

$$
\begin{aligned}
\left\|A x\left(t_{2}\right)-A x\left(t_{1}\right)\right\| & =\phi\left(A x\left(t_{2}\right)-A x\left(t_{1}\right)\right) \leq\left|\phi\left(p\left(t_{2}\right)-p\left(t_{1}\right)\right)\right| \\
& +\left|\int_{0}^{t_{2}} \phi(f(s, x(s))) d_{s} g\left(t_{2}, s\right)-\int_{0}^{t_{1}} \phi(f(s, x(s))) d_{s} g\left(t_{1}, s\right)\right| \\
& \leq\left\|p\left(t_{2}\right)-p\left(t_{1}\right)\right\|+\mid \int_{0}^{t_{1}} \phi(f(s, x(s))) d_{s} g\left(t_{2}, s\right) \\
& +\int_{t_{1}}^{t_{2}} \phi(f(s, x(s))) d_{s} g\left(t_{2}, s\right)-\int_{0}^{t_{1}} \phi(f(s, x(s))) d_{s} g\left(t_{1}, s\right) \mid \\
& \leq\left\|p\left(t_{2}\right)-p\left(t_{1}\right)\right\|+\left|\int_{0}^{t_{1}} \phi(f(s, x(s))) d_{s}\left[g\left(t_{2}, s\right)-g\left(t_{1}, s\right)\right]\right| \\
& +\left|\int_{t_{1}}^{t_{2}} \phi(f(s, x(s))) d_{s} g\left(t_{2}, s\right)\right|
\end{aligned}
$$




$$
\begin{aligned}
& \leq \quad\left\|p\left(t_{2}\right)-p\left(t_{1}\right)\right\| \\
& +\quad \int_{0}^{t_{1}}|\phi(f(s, x(s)))| d_{s}\left[\bigvee_{z=0}^{s}\left(g\left(t_{2}, z\right)-g\left(t_{1}, z\right)\right)\right] \\
& +\quad \int_{t_{1}}^{t_{2}}|\phi(f(s, x(s)))| d_{s}\left[\bigvee_{z=0}^{s} g\left(t_{2}, z\right)\right] \\
& \leq \quad\left\|p\left(t_{2}\right)-p\left(t_{1}\right)\right\|+M \int_{0}^{t_{1}} d_{s}\left[\bigvee_{z=0}^{s}\left(g\left(t_{2}, z\right)-g\left(t_{1}, z\right)\right)\right] \\
& +\quad M \int_{t_{1}}^{t_{2}} d_{s}\left[\bigvee_{z=0} g\left(t_{2}, z\right)\right] \\
& \leq \quad\left\|p\left(t_{2}\right)-p\left(t_{1}\right)\right\|+M \bigvee_{s=0}^{t_{1}}\left(g\left(t_{2}, s\right)-g\left(t_{1}, s\right)\right) \\
& +\quad M\left[\bigvee_{s=0}^{t_{2}} g\left(t_{2}, s\right)-\bigvee_{s=0}^{t_{1}} g\left(t_{2}, s\right)\right] \\
& \leq \quad\left\|p\left(t_{2}\right)-p\left(t_{1}\right)\right\|+M N(\epsilon)+M \bigvee_{s=t_{1}}^{t_{2}} g\left(t_{2}, s\right)
\end{aligned}
$$

where

$$
N(\epsilon)=\sup \left\{\bigvee_{s=0}^{t_{1}}\left(g\left(t_{2}, s\right)-g\left(t_{1}, s\right)\right): t_{1}, t_{2} \in I, t_{1}<t_{2}, t_{2}-t_{1} \leq \epsilon\right\}
$$

Hence

$$
\left\|A x\left(t_{2}\right)-A x\left(t_{1}\right)\right\| \leq\left\|p\left(t_{2}\right)-p\left(t_{1}\right)\right\|+M N(\epsilon)+M \bigvee_{s=t_{1}}^{t_{2}} g\left(t_{2}, s\right)
$$

and so $A x \in C[I, E]$. We claim that $A: Q \rightarrow Q$ is weakly sequentially continuous and $A(Q)$ is weakly relatively compact. Once the claim is established, Theorem 1.5 guarantees the existence of a fixed point $x \in C[I, E]$ of the operator $A$ and the integral equation (2.5) has a solution $x \in C[I, E]$.

To prove our claim, we start by showing that $A: Q \rightarrow Q$. Take $x \in Q$, note that the inequality (2.6) shows that $A Q$ is norm continuous. Then by using Proposition 1.8 
we get

$$
\begin{aligned}
\|A x(t)\| & =\phi(A x(t)) \leq|\phi(p(t))|+\left|\phi\left(\int_{0}^{t} f(s, x(s)) d_{s} g(t, s)\right)\right| \\
& \leq\|p(t)\|+\int_{0}^{t}|\phi(f(s, x(s)))| d_{s}\left(\bigvee_{z=0}^{s} g(t, z)\right) \\
& \leq\|p(t)\|+M \int_{0}^{t} d_{s}\left(\bigvee_{z=0}^{s} g(t, z)\right) \\
& \leq\|p(t)\|+M \bigvee_{s=0}^{t} g(t, s) \\
& \leq\|p\|_{0}+M \sup _{t \in I} \bigvee_{s=0}^{t} g(t, s) \\
& \leq\|p\|_{0}+M K=M_{0} .
\end{aligned}
$$

Then

$$
\|A x\|_{0}=\sup _{t \in I}\|A x(t)\| \leq M_{0}
$$

Hence, $A x \in Q$ and $A Q \subset Q$ which prove that $A: Q \rightarrow Q$, and $A Q$ is bounded in $C[I, E]$.

We need to prove now that $A: Q \rightarrow Q$ is weakly sequentially continuous. Let $\left\{x_{n}(t)\right\}$ be sequence in $Q$ weakly convergent to $x(t)$ in $E$, since $Q$ is closed we have $x \in Q$. Fix $t \in I$, since $f$ satisfies (ii), then we have $f\left(t, x_{n}(t)\right)$ converges weakly to $f(t, x(t))$. By the Lebesgue dominated convergence theorem (see assumption (iii)) for Pettis integral ([12]), we have for each $\phi \in E^{*}, s \in I$

$$
\begin{aligned}
\phi\left(\int_{0}^{t} f\left(s, x_{n}(s)\right) d_{s} g(t, s)\right)= & \int_{0}^{t} \phi\left(f\left(s, x_{n}(s)\right)\right) d_{s} g(t, s) \\
& \rightarrow \int_{0}^{t} \phi(f(s, x(s))) d_{s} g(t, s), \forall \phi \in E^{*}, t \in I,
\end{aligned}
$$

i.e. $\phi\left(A x_{n}(t)\right) \rightarrow \phi(A x(t)), \forall t \in I, A x_{n}(t)$ converging weakly to $A x(t)$ in $E$.

Thus, $A$ is weakly sequentially continuous on $Q$.

Next we show that $A Q(t)$ is relatively weakly compact in $E$.

Note that $Q$ is nonempty, closed, convex and uniformly bounded subset of $C[I, E]$ and $A Q$ is bounded in norm. According to Propositions 1.6 and 1.7, $A Q$ is relatively weakly compact in $C[I, E]$ implies $A Q(t)$ is relatively weakly compact in $E$, for each $t \in I$.

Since all conditions of Theorem 1.5 are satisfied, then the operator $A$ has at least one fixed point $x \in Q$ and the nonlinear Stieltjes integral equation (2.5) has at least one weak solution $x \in C[I, E]$. 


\section{Volterra integral equation of fractional order}

In this section we show that the Volterra integral equation of fractional order

$$
x(t)=p(t)+\int_{0}^{t} \frac{(t-s)^{\alpha-1}}{\Gamma(\alpha)} f(s, x(s)) d s, t \in I
$$

can be considered as a special case of the Volterra-Stieltjes integral equation (2.1), where the integral is in the sense of weakly Riemann.

First, consider, as previously, that the function $g(t, s)=g: \Lambda \rightarrow R$. Moreover, we will assume that the function $g$ satisfies the following condition

(vi') For $t_{1}, t_{2} \in I, t_{1}<t_{2}$, the function $s \rightarrow g\left(t_{2}, s\right)-g\left(t_{1}, s\right)$ is nonincreasing on $\left[0, t_{1}\right]$.

Now, we have the following lemmas which proved by Banaś et al. [5].

Lemma 3.1. Under assumptions (vi') and (vii), for any fixed $s \in I$, the function $t \rightarrow$ $g(t, s)$ is nonincreasing on $[s, 1]$.

Lemma 3.2. Under assumptions (iv), (vi') and (vii), the function $g$ satisfies assumption (vi).

Consider the function $g$ defined by

$$
g(t, s)=\frac{t^{\alpha}-(t-s)^{\alpha}}{\Gamma(\alpha+1)} .
$$

Now, we show that the function $g$ satisfies assumptions (iv), (v), (vi') and (vii). Clearly that the function $g$ satisfies assumptions (iv) and (vii). Also we get

$$
d_{s} g(t, s)=\frac{(t-s)^{\alpha-1}}{\Gamma(\alpha)}>0
$$

for $0 \leq s<t$. This implies that $s \rightarrow g(t, s)$ is increasing on $[0, t]$ for any fixed $t \in I$. Thus the function $g$ satisfies assumption (v).

To show that $g$ satisfies assumption ( $\left.\mathrm{vi}^{\prime}\right)$, let us fix arbitrary $t_{1}, t_{2} \in[0, T], t_{1}<t_{2}$. Then we get

$$
G(s)=g\left(t_{2}, s\right)-g\left(t_{1}, s\right)=\frac{t_{2}^{\alpha}-t_{1}^{\alpha}-\left(t_{2}-s\right)^{\alpha}+\left(t_{1}-s\right)^{\alpha}}{\Gamma(\alpha+1)},
$$

define on $\left[0, t_{1}\right]$. Thus

$$
G^{\prime}(s)=\frac{\left(t_{2}-s\right)^{\alpha-1}-\left(t_{1}-s\right)^{\alpha-1}}{\Gamma(\alpha)}=\frac{1}{\Gamma(\alpha)}\left[\frac{1}{\left(t_{2}-s\right)^{1-\alpha}}-\frac{1}{\left(t_{1}-s\right)^{1-\alpha}}\right] .
$$

Hence $G^{\prime}(s)<0$ for $s \in\left[0, t_{1}\right)$. This means that $g$ satisfies assumption (vi'). And the function $g$ satisfies assumptions (iv)-(vii) in Theorem 2.5.

Hence, the equation (3.7) can be written in the form

$$
x(t)=p(t)+\int_{0}^{t} f(s, x(s)) d_{s} g(t, s) .
$$


And the equation (3.7) is a special case of the equation (2.5).

Now, we estimate the constants $K, N(\epsilon)$ used in our proof. To see this, since the function $s \rightarrow g(t, s)$ is nondecreasing on $[0, t]$ for any fixed $t \in I$. Then we have

$$
\bigvee_{s=0}^{t} g(t, s)=g(t, t)-g(t, 0)=g(t, t)=\frac{t^{\alpha}}{\Gamma(\alpha+1)},
$$

and

$$
\begin{aligned}
\bigvee_{s=0}^{t_{1}}\left(g\left(t_{2}, s\right)-g\left(t_{1}, s\right)\right) & =\sum_{i=1}^{n}\left|\left[g\left(t_{2}, s_{i}\right)-g\left(t_{1}, s_{i}\right)\right]-\left[g\left(t_{2}, s_{i-1}\right)-g\left(t_{1}, s_{i-1}\right)\right]\right| \\
& =\sum_{i=1}^{n}\left\{\left[g\left(t_{2}, s_{i-1}\right)-g\left(t_{1}, s_{i-1}\right)\right]-\left[g\left(t_{2}, s_{i}\right)-g\left(t_{1}, s_{i}\right)\right]\right\} \\
& =g\left(t_{1}, t_{1}\right)-g\left(t_{2}, t_{1}\right) \\
& =\frac{1}{\Gamma(\alpha+1)}\left[t_{1}^{\alpha}-t_{2}^{\alpha}+\left(t_{2}-t_{1}\right)^{\alpha}\right] .
\end{aligned}
$$

Thus

$$
K=\sup \left\{\bigvee_{s=0}^{t} g(t, s): t \in I\right\}=\frac{T^{\alpha}}{\Gamma(\alpha+1)}
$$

and

$$
\begin{gathered}
N(\epsilon)=\sup \left\{\bigvee_{s=0}^{t_{1}}\left(g\left(t_{2}, s\right)-g\left(t_{1}, s\right)\right): t_{1}, t_{2} \in I, t_{1}<t_{2}, t_{2}-t_{1} \leq \epsilon\right\} \\
=\frac{1}{\Gamma(\alpha+1)}\left[t_{1}^{\alpha}-t_{2}^{\alpha}+\left(t_{2}-t_{1}\right)^{\alpha}\right]
\end{gathered}
$$

Since

$$
\begin{aligned}
\bigvee_{s=t_{1}}^{t_{2}} g\left(t_{2}, s\right) & =g\left(t_{2}, t_{2}\right)-g\left(t_{2}, t_{1}\right) \\
& =\frac{1}{\Gamma(\alpha+1)}\left[t_{2}^{\alpha}-\left(t_{2}-t_{2}\right)^{\alpha}-t_{2}^{\alpha}+\left(t_{2}-t_{1}\right)^{\alpha}\right] \\
& =\frac{\left(t_{2}-t_{1}\right)^{\alpha}}{\Gamma(\alpha+1)} .
\end{aligned}
$$

Then

$$
\begin{gathered}
Q=\left\{x \in C[I, E]:\|x\|_{0} \leq M_{0}\right. \\
\left.\left\|x\left(t_{2}\right)-x\left(t_{1}\right)\right\| \leq\left\|p\left(t_{2}\right)-p\left(t_{1}\right)\right\|+\frac{M}{\Gamma(\alpha+1)}\left[\left|t_{1}^{\alpha}-t_{2}^{\alpha}\right|+2\left(t_{2}-t_{1}\right)^{\alpha}\right]\right\} .
\end{gathered}
$$

Finally, we can formulate the following existence result concerning the fractional integral equation (3.7).

Theorem 3.3. Under the assumptions (i)-(iii), the fractional integral equation (3.7) has at least one weak solution $x \in C[I, E]$. 


\section{Fractional differential equations}

In this section we establish existence results for the fractional differential equations (1.1)-(1.2) and (1.3)-(1.4) in the reflexive Banach space $E$.

\subsection{Weak solution}

Consider the integral equation

$$
x(t)=\int_{0}^{t} \frac{(t-s)^{\alpha-1}}{\Gamma(\alpha)} f(s, x(s)) d s, t \in I,
$$

where the integral is in the sense of weakly Riemann.

Lemma 4.1. Let $\alpha \in(0,1)$. A function $x$ is a weak solution of the fractional integral equation (4.9) if and only if $x$ is a solution of the problem (1.1)-(1.2).

Proof. Integrating (1.1)-(1.2) we obtain the integral equation (4.9). Operating by ${ }_{R} D^{\alpha}$ on (4.9) we obtain the problem (1.1)-(1.2). So the equivalent between (1.1)(1.2) and the integral equation (4.9) is proved and then the results follows from Theorem 3.3.

\subsection{Mild solution}

Consider now the problem (1.3)-(1.4). According to Definitions 1.1 and 1.3 , it is suitable to rewrite the problem (1.3)-(1.4) in the integral equation

$$
x(t)=x_{0}+\int_{0}^{t} \frac{(t-s)^{\alpha-1}}{\Gamma(\alpha)} f(s, x(s)) d s, t \in I .
$$

Definition 4.2. By the mild solution of the problem (1.3)-(1.4), we mean that the function $x \in C[I, E]$ which satisfies the corresponding integral equation of (1.3)-(1.4) which is $(4.10)$.

Theorem 4.3. If (i)-(iii) are satisfied, then the problem (1.3)-(1.4) has at least one mild solution $x \in C[I, E]$.

It is often the case that the problem (1.3)-(1.4) does not have a differentiable solution yet does have a solution, in a mild sense.

\section{References}

[1] J. Banaś, Some properties of Urysohn-Stieltjes integral operators, Internat. J. Math. and Math. Sci. 21 (1998) 79-88.

[2] J. Banaś, K. Sadarangani, Solvability of Volterra-Stieltjes operator-integral equations and their applications, Comput. Math. Appl. 4112 (2001) 1535-1544. 
[3] J. Banaś, J.C. Mena, Some properties of nonlinear Volterra-Stieltjes integral operators, Comput. Math. Appl. 49 (2005) 1565-1573.

[4] J. Banaś, D. O'Regan, Volterra-Stieltjes integral operators, Math. Comput. Modelling. 41 (2005) 335-344.

[5] J. Banaś, T. Zając, A new approach to the theory of functional integral equations of fractional order, J. Math. Anal. Appl. 375 (2011) 375-387.

[6] M. Benchohra, F. Mostefai, Weak solutions for nonlinear fractional differential equations with integral boundary conditions in Banach spaces, Opuscula Mathematica 321 (2012) 31-40.

[7] M. Benchohra, J.R. Graef and F. Mostefai, Weak solutions for nonlinear fractional differential equations on reflexive Banach spaces, Electron. J. Qual. Theory Differ. Equ. 54 (2010) 1-10.

[8] C.W. Bitzer, Stieltjes-Volterra integral equations, Illinois J. Math. 14 (1970) 434451.

[9] M. Caputo, Linear models of dissipation whose $Q$ is almost frequency independent-II, Geophys. J.R. Astr. Soc. 13 (1967) 529-539.

[10] N. Dunford, J.T. Schwartz, Linear Operators, Interscience, Wiley, New York 1958.

[11] A.M.A. El-Sayed, W.G. El-Sayed and A.A.H. Abd El-Mowla, Volterra-Stieltjes integral equation in reflexive Banach spaces, Electronic Journal of Mathematical Analysis and Applications 51 (2017) 287-293.

[12] R.F. Geitz, Pettis integration, Proc. Amer. Math. Soc. 82 (1981) 81-86.

[13] E. Hille, R.S. Phillips, Functional Analysis and Semi-groups, Amer. Math. Soc. Colloq. Publ. Providence, R. I. 1957.

[14] H.H.G. Hashem, Weak solutions of differential equations in Banach spaces, Journal of Fractional Calculus and Applications 31 (2012) 1-9.

[15] T. Margulies, Wave propagation in viscoelastic horns using a fractional calculus rheology model, The Journal of the Acoustical Society of America 1142442 (2003), https://doi.org/10.1121/1.4779280.

[16] B. Mathieu, P. Melchior, A. Oustaloup and Ch. Ceyral, Fractional differentiation for edge detection, Fractional Signal Processing and Applications 83 (2003) 22852480 .

[17] A.R. Mitchell, Ch. Smith, An existence theorem for weak solutions of differential equations in Banach spaces, Nonlinear Equations in Abstract Spaces (V. Lakshmikantham, ed.) (1978) 387-404. 
[18] I. Podlubny, Fractional Differential Equations, Academic Press, New York 1999.

[19] D. O'Regan, Fixed point theory for weakly sequentially continuous mapping, Math. Comput. Modeling 27 (1998) 1-14.

[20] A. Szep, Existence theorem for weak solutions of ordinary differential equations in reflexive Banach spaces, Studia Sci. Math. Hungar. 6 (1971) 197-203.

[21] S.G. Samko, A.A. Kilbas and O. Marichev, Integral and Derivatives of Fractional Orders and Some of Their Applications, Nauka i Teknika, Minsk 1987.

[22] H.A.H. Salem, A.M.A. El-Sayed, Weak solution for fractional order integral equations in reflexive Banach spaces, Math. Slovaca 55 (2005) 169-181.

[23] H.A.H. Salem, A.M.A. El-Sayed, A note on the fractional calculus in Banach spaces, Studia Sci. Math. Hungar. 422 (2005) 115-130.

[24] H.A.H. Salem, Quadratic integral equations in reflexive Banach space, Discuss. Math. Differ. Incl. Control Optim. 30 (2010) 61-69.

DOI: $10.7862 / \mathrm{rf} .2017 .6$

\section{Ahmed M.A El-Sayed}

email: amasayed@alexu.edu.eg

Faculty of Science

Alexandria University

Alexandria

EGYPT

Wagdy G. El-Sayed

email: wagdygoma@alexu.edu.eg

Faculty of Science

Alexandria University

Alexandria

EGYPT

\section{A.A.H. Abd El-Mowla}

email: aziza.abdelmwla@yahoo.com

Faculty of Science

Omar Al-Mukhtar University

Derna

LIBYA

Received 1.03.2017

Accepted 30.10.2017 